SHORT REPORT

\title{
Progression of cerebral white matter lesions in Alzheimer's disease: a new window for therapy?
}

\author{
F-E de Leeuw, F Barkhof, P Scheltens
}

See Editorial Commentary, p 1185

J Neurol Neurosurg Psychiatry 2005;76:1286-1288. doi: 10.1136/jnnp.2004.053686

Background: White matter lesions (WML) are a risk factor for Alzheimer's disease. Progression of WML is associated with vascular factors and cognitive decline in population based studies but the course of WML is unknown in Alzheimer's disease.

Objective: To investigate the prevalence and risk factors for progression of WML in Alzheimer's disease.

Subjects: 38 patients with Alzheimer's disease for whom blood pressure measurements and sequential brain MRIs were available.

Methods: The proportion of patients with progression of WML was calculated, stratified on baseline absence or presence of WML by analysis of variance. Odds ratios (OR) were calculated by age and sex adjusted logistic regression to quantify the relation between blood pressure and progression of WML.

Results: About $25 \%$ of the patients showed progression of WML. Patients with WML at baseline had significantly more progression than those without WML at baseline (adjusted mean difference $=1.2 ; 95 \%$ confidence interval $(\mathrm{Cl}), 0.6$ to 1.8). Diastolic blood pressure (DBP) was particularly related to progression of WML $(O R=5.9(95 \% \mathrm{Cl}, 1.0$ to 37.6$)$ per $10 \mathrm{~mm} \mathrm{Hg}$ DBP, $p=0.05$ ).

Conclusions: Alzheimer's disease patients with WML at baseline are at risk for rapid progression of WML. WML may offer a potential treatment target in this disease to ameliorate the rate of cognitive decline.

$\mathrm{T}$ here is evidence that subcortical vascular white matter lesions (WML) result in an increased risk for Alzheimer's disease. ${ }^{1}$ In population based studies, individuals with progression of WML experience a steeper decline in cognition than those without. Identification of progression of WML in the course of Alzheimer's disease would provide further evidence for a causal role for vascular factors in this disorder. However, neither the degree of progression of WML nor the identification of risk factors for progression have yet been studied in this disease. Proof of progression would offer the possibility of identifying a subpopulation of Alzheimer patients in whom further cognitive decline may be ameliorated by treatment of a potentially modifiable vascular risk factor. With this in mind we set out to investigate the prevalence and risk factors of progression of WML in Alzheimer's disease.

\section{METHODS}

In the study period, 268 consecutive patients were seen at the Alzheimer Centre of the VU Medical Centre, Amsterdam, with "probable" Alzheimer's disease according to the NINCDS-ADRDA criteria. Of these, 38 had sequential brain magnetic resonance imaging (MRI), with an initial scan as part of a routine diagnostic procedure (baseline) and a follow up scan as part of a routine outpatient follow up. These 38 patients formed the study group. The mean follow up period was 2.2 years (range 1.0 to 5.1), and did not differ between those with and without progression of white matter lesions.

All patients were examined using a fluid attenuated inversion recovery (FLAIR) pulse sequence on a $1.0 \mathrm{~T}$ scanner (Impact, Siemens, Erhlangen, Germany). WML were rated using the age related white matter changes (ARWMC) rating scale. $^{2}$ Briefly, WML were rated on a four point scale (0-3) in five regions in the left and right hemisphere separately, leading to an ARWMC sum score (range 0-30). Baseline and follow up scans were rated independently, blinded for scan order and clinical data. Intrarater studies on a random sample of 32 of the 38 scans showed good to excellent agreement $(\kappa=0.6$ to 1.0$)$.

Blood pressure was measured with a mercury sphygmomanometer and with the participant in a sitting position after at least five minutes of rest. Self reported or informant report on smoking behaviour (ever/never) was recorded from the patient's clinical history. Diabetes mellitus was considered to be present if the participant was taking glucose lowering drugs or if the random glucose was higher than $11.1 \mathrm{mmol} / \mathrm{l}$. Global cognitive function was assessed by the mini-mental state examination (MMSE).

Progression of WML was defined as an increase of one point or more on the ARWMC scale between baseline and follow up (fig 1). We calculated the proportion of patients with progression of WML stratified on sex and on baseline absence or presence of WML by analysis of variance (ANOVA). In addition we calculated the mean diastolic and systolic blood pressure (DBP and SBP) in patients with or without progression of WML by ANOVA. We calculated odds ratios (OR) by age and sex adjusted logistic regression to quantify the relation between blood pressure and the progression of WML. Additional adjustments were made for possible confounding factors, including smoking (ever/ never), diabetes mellitus (yes/no), and global cognitive function.

\section{RESULTS}

Patients with a follow up scan were older than those without (mean (SD) age, $72.2(9.4)$ years $v 65.9(8.8)$ years $(\mathrm{p}<0.05)$ ). Mean MMSE score at baseline was 21.4. Other baseline characteristics are presented in table 1. Except for age, there were no differences in baseline characteristics between those with or without follow up MRI.

Mean ARWMC score was 0.8 at baseline (median 0; interquartile range 0 to 0.25 ) and 1.5 during follow up

Abbreviations: $A R W M C$, age related white matter changes rating scale; MMSE, mini-mental state examination; NINCDS-ADRDA, National Institute of Neurological and Communicative DisordersAlzheimer's Disease and Related Disorders Association; WML, white matter lesion 


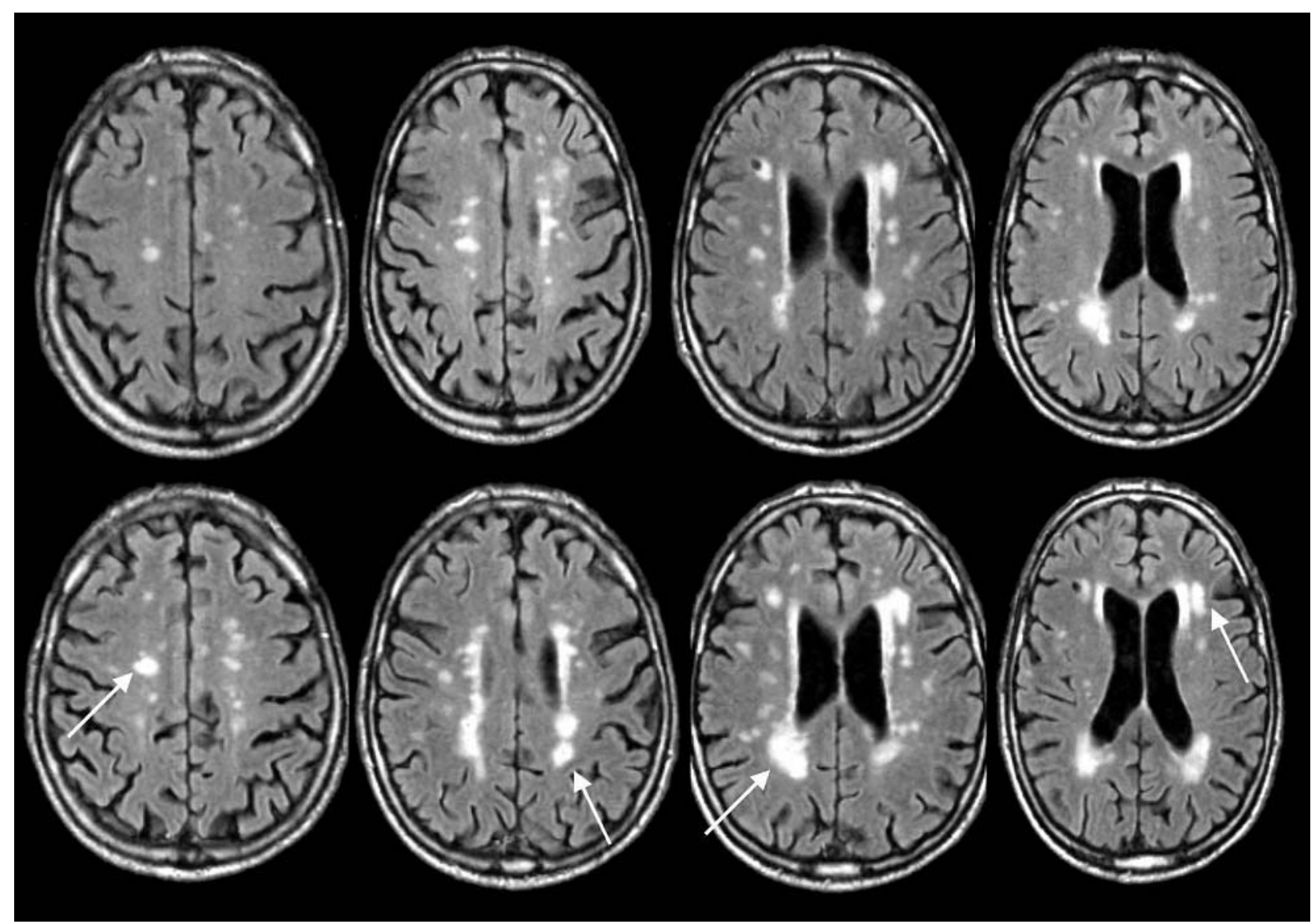

Figure 1 Axial (transverse) slices at four different sections of the brain at baseline (upper panel; mean age related white matter changes rating scale $($ ARWMC) score $=1$ ) and during follow up (lower panel; mean ARWMC score $=5$ ) in a 79 year old male patient with Alzheimer's disease and progression of white matter lesions (WML). Arrows indicate some examples of emergence or progression of WML.

(median 0; interquartile range 0 to 1.25 ). About $25 \%$ of the patients showed progression of WML. The relative risk of age for progression of WML was 1.2 per year (95\% confidence interval (CI), 1.1 to 1.5$)$. Men had more progression of WML than women $(30.0 \% \vee 16.7 \%, \mathrm{p}=0.34)$, especially above 70 years $(55.6 \% \vee 40.0 \%, \mathrm{p}=0.02)$. Patients with WML at baseline had significantly more progression of WML than

Table 1 Characteristics of the study population at baseline, with or without serial magnetic resonance imaging

\begin{tabular}{lll}
\hline Characteristic & With follow up & $\begin{array}{l}\text { Without } \\
\text { follow up }\end{array}$ \\
\hline Number of subjects & 38 & 230 \\
Age at baseline (years) & $65.9(8.8)$ & $72.3(9.7)^{*}$ \\
Women & $47.4 \%$ & $60.9 \%$ \\
Mean duration of follow up (years; & $2.2(1.0$ to 5.1) na \\
range) & $0.8(0$ to 8$)$ & $1.8(3.7)$ \\
Mean ARWMC baseline (range) & $1.5(0$ to 17) & na \\
Mean ARWMC follow up & $78.4 \%$ & $57.2 \%$ \\
Hypertension & $149.5(25.8)$ & $150.5(21.9)$ \\
Systolic blood pressure (mm Hg) & $85.3(12.3)$ & $86.0(10.5)$ \\
Diastolic blood pressure (mm Hg) & 0 & $9.4 \%$ \\
Diabetes & $32.1 \%$ & $37.8 \%$ \\
Smoking (ever) & $21.4(4.6)$ & $19.7(5.3)$ \\
MMSE & \multicolumn{3}{l}{} \\
\hline Values are age and sex adjusted means (SD) or percentages unless stated \\
otherwise.
\end{tabular}

those without (adjusted mean difference in ARWMC score 1.2 (95\% CI, 0.6 to 1.8 ); $\mathrm{p}<0.05$ ). Patients with progression had a higher DBP than those without (92.9 (14.9) $v 82.8$ (11.1) $\mathrm{mm} \mathrm{Hg}(\mathrm{p}=0.05))$. This was not true for SBP.

The relative risk of baseline SBP for progression of WML was 1.6 per $10 \mathrm{~mm} \mathrm{Hg}$ increase in blood pressure (95\% CI, 0.9 to 2.8) $(\mathrm{p}=0.12)$ and the relative risk of baseline DBP was 5.9 (1.0 to 37.6) $(\mathrm{p}=0.05)$. Adjustment for confounding factors did not alter the magnitude of the association.

\section{DISCUSSION}

Our study shows that over the relatively short period of two years WML load doubled in a sample of typical Alzheimer's disease patients. The risk of progression was influenced by age, sex, baseline ARWMC score, and DBP.

Selection bias may have influenced our findings. As blood pressure is the most important risk factor for $\mathrm{WML}^{3}$ it is possible that patients with the highest degree of WML were unable to visit an outpatient clinic any more-for example because of gait disturbances. ${ }^{4}$ However, this selection bias presumably only played a role in patients with the most severe WML and hypertension; thus this form of bias may even have underestimated progression of WML. Because only single blood pressure measurements were available to us, misclassification of blood pressure might have occurred; however, as these measurements were done without prior notice of the neuroimaging findings, any misclassification would have been random and not related to the outcome. As such, it might even have diluted (underestimated) the effects that we found. 
We measured WML using the ARWMC scale, which is an ordinal scale with a predefined maximum score. This may limit the detectability of changes in WML: the change has to be of a certain magnitude in order to result in a one point increase on the ARWMC scale; furthermore, this scale has a ceiling effect, in contrast to volumetric methods. This type of measurement error is most likely to have affected those patients with the highest degree of WML, presumably even leading to an underestimation of the relative risk of blood pressure on the progression of WML.

Our findings suggest that Alzheimer's disease patients with WML at baseline are at risk for rapid progression of WML. This is in line with findings from population based studies. ${ }^{5}$ They may therefore be at increased risk of progressive cognitive decline compared with patients without baseline WML. If these findings are substantiated, it could indicate that further cognitive decline in Alzheimer patients might be ameliorated by modifying the progression of WML-for example by treating hypertension. Future studies should address whether progression of WML coincides with cognitive decline, and whether an intervention can modify this favourably. ${ }^{6}$

\section{Authors' affiliations}

P Scheltens, Alzheimer Centre and Department of Neurology, VU

Medical Centre, Amsterdam, Netherlands
F Barkhof, Department of Radiology VU Medical Centre

F-E de Leeuw, Department of Neurology, University Medical Centre St Radboud, Niimegen, Netherlands

Competing interests: none declared

Correspondence to: Dr F-E de Leeuw, Department of Neurology (HP326), University Medical Centre St Radboud, PO Box 9101, 6500HB Nijmegen, Netherlands; h.deleeuw@neuro.umcn.nl

Received 9 September 2004

In revised form 6 February 2005

Accepted 15 February 2005

\section{REFERENCES}

1 Vermeer SE, Prins ND, den Heijer T, et al. Silent brain infarcts and the risk of dementia and cognitive decline. N Engl J Med 2003;348:1215-22.

2 Wahlund LO, Barkhof $F$, Fazekas $F$, et al. A new rating scale for age-related white matter changes applicable to MRI and CT. Stroke 2001;32:1318-22.

3 De Leeuw FE, de Groot JC, Oudkerk M, et al. Hypertension and cerebral white matter lesions in a prospective cohort study. Brain 2002;125:765-72.

4 Benson RR, Guttmann CR, Wei X, et al. Older people with impaired mobility have specific loci of periventricular abnormality on MRI. Neurology 2002; 58:48-55.

5 Schmidt R, Enzinger C, Ropele S, et al. Progression of cerebral white matter lesions: 6-year results of the Austrian Stroke Prevention Study. Lancet 2003;361:2046-8.

6 Schmidt R, Scheltens P, Erkinjuntti T, for the European Task Force on AgeRelated White Matter Changes, et al. White matter lesion progression: a surrogate endpoint for trials in cerebral small-vessel disease. Neurology 2004;63:139-44.

\section{Call for papers}

$11^{\text {th }}$ European Forum on Quality Improvement in Health Care 26-28 April 2006, Prague, Czech Republic Deadline 30 September 2005.

For further information and to submit online go to: www.quality.bmjpg.com 